\title{
CARACTERIZAÇÃO MORFOLÓGICA E DISTRIBUIÇÃO TEMPORAL DE LARVAS E JUVENIS DE APAREIODON AFFINIS (STEINDACHNER) (OSTEICHTHYES, PARODONTIDAE) NO ALTO RIO PARANÁ, PARANÁ
}

\author{
Andréa Bialetzki ${ }^{1}$ \\ Paulo Vanderlei Sanches ${ }^{1}$ \\ Gilmar Baumgartner ${ }^{1}$ \\ Keshiyu Nakatani ${ }^{1}$
}

\begin{abstract}
MORPHOLOGICAL CHARACTERIZATION AND TEMPORAL DISTRIBUTION OF LARVAE AND JUVENILES OF APAREIOdON afFINIS (STEINDACHNER) (OSTEICHTHYES, PA. RODONTIDAE) IN UPPER PARANÁ RIVER, PARANÁ. With the purpose of characterizing morphologicaly the larvae and juveniles of Apareiodon affinis (Steindachner, 1879) and to verify their temporal distribution monthly samples were taken at Leopoldo's Inlet, upper Paraná River. The specimens were captured with a conic-cylindrical plankton net of mesh size 0,5mm from March 1991 to February 1992. Soon after identification, the specimens were separeted according to their development (preflexion, flexion, posflexion and juvenile) and then the morphometric analyses were performed. The results showed that the larvae were captured from August 1991 to February 1992, with greatest densities in November 1991. The specimens occured in every timetable, however, larvae in preflexion and flexion were captured during day time and, posflexion and juvenile, at night. According to series of morphological development, high body proportions variation were verifyed mainly in the head lenght, eye diameter and in height body. Moreover, the individuals showed little pigmentation and myomeres number changing between 39 to 42 . The majority of morphometric characteristics have negative allometry with the exception of head lenght with regard to standart lenght that have positive allometry.
\end{abstract}

KEY WORDS. Apareiodon affinis, morphological development, temporal distribution, upper Paraná River

O entendimento da dinâmica reprodutiva da comunidade íctica e suas relações com o meio é auxiliado pelo conhecimento das áreas e períodos de desova. Entretanto, um dos maiores obstáculos encontrados nesta área de estudo é a identificação das larvas coletadas em ambiente natural. Estudos taxonômicos são limitados principalmente pela grande similaridade morfológica encontrada nos primeiros estágios de desenvolvimento das diferentes espécies (SNYDER 1981; FUIMAN et al. 1983), e pela falta de descrições adequadas e chaves ou guias apropriadas para este fim.

1) Departamento de Biologia/Nupélia, Curso de Pós-Graduação em Ecologia de Ambientes Aquáticos Continentais, Universidade Estadual de Maringá. Avenida Colombo 5790, 87020-900 Maringá, Paraná, Brasil.

E-mail: agostinhoaa@wnet.com.br

Revta bras. Zool. 15 (4): 1037 - 1047, 1998 
Apareiodon affinis (Steindachner, 1879), vulgarmente conhecido como "canivete", apresenta grande distribuição geográfica sendo freqüente nas bacias dos rios Paraná, Paraguai e Uruguai (GoDoY 1975). Apresenta ainda uma grande flexibilidade de habitats, sendo comum encontrá-lo em rios, riachos, águas represadas e lagoas. Em virtude do seu pequeno porte (até $15 \mathrm{~cm}$ ), a espécie não apresenta interesse comercial, sendo utilizada apenas como isca para pesca profissional e amadora, entretanto, ecologicamente, é extremamente importante, uma vez que serve como elo de transferência de energia para níveis mais elevados da cadeia trófica. Quanto à reprodução, é caracterizada por possuir desova do tipo parcelada e sazonal, não apresentando cuidado com a prole (VAZZOLER 1996).

Estudos relativos às características morfológicas de larvas dessa espécie ficam restritos àquele realizado por OLDANI (1977) no médio rio Paraná. Para o alto rio Paraná pouco se sabe sobre seu desenvolvimento larval. Desta forma, este trabalho tem por objetivo apresentar algumas características morfológicas, baseadas em caracteres morfométricos e merísticos, observadas em larvas e juvenis, bem como analisar a distribuição temporal desses estágios.

\section{MATERIAL E MÉTODOS}

Os espécimes analisados foram obtidos mensalmente durante o período de março de 1991 a fevereiro de 1992, no ressaco do Leopoldo, margem direita da ilha Porto Rico, alto rio Paraná, município de Porto Rico, Paraná (Fig. 1). As coletas foram realizadas durante ciclos nictemerais com intervalos de quatro horas entre as amostragens, utilizando-se rede de plâncton do tipo cônico-cilíndrica de malha $0,5 \mathrm{~mm}$ e fluxômetro acoplado à boca para obtenção do volume de água filtrada, a qual foi arrastada por 10 minutos com o barco à baixa velocidade. As amostras obtidas foram fixadas em formol $4 \%$ tamponado com carbonato de cálcio.

Após a separação do restante do plâncton, o material foi identificado seguindo a técnica de sequência de desenvolvimento proposta por AHLSTROM \& MOSER (1976). Foram analisados 153 indivíduos com comprimento padrão variando de $4,46 \mathrm{~mm}$ a $24,00 \mathrm{~mm}$, que foram separados de acordo com o desenvolvimento (pré-flexão, flexão, pós-flexão e juvenil) segundo terminologia descrita por AHLSTROM \& BALL (1954) e KENDALL et al. (1984).

A descrição de cada estágio foi baseada no grau de desenvolvimento e na ocorrência dos principais eventos morfológicos, sendo as ilustrações feitas com auxílio de câmara clara. Para cada indivíduo foram tomadas através de um microscópico estereoscópico equipado com ocular micrométrica, as seguintes variáveis morfométricas: a) comprimento padrão (CP) - distância da ponta do focinho ao final da notocorda; b) comprimento da cabeça (CC) - distância entre a ponta do focinho e o limite posterior da abertura branquial; c) altura do corpo (ACO) - distância máxima entre os perfis dorsal e ventral do corpo; d) diâmetro do olho - (DOL) diâmetro da área pigmentada medida longitudinalmente; e) comprimento do focinho - (CF) distância entre a ponta do focinho e margem anterior do olho; f) altura da cabeça - (AC) distância dorso-ventral perpendicular máxima ao eixo longitudinal do corpo; g) distância pré-dorsal (FND) - distância entre a ponta do focinho e a 
tangente vertical da margem anterior da nadadeira dorsal; $h$ ) distância pré-anal (FNA) - distância entre a ponta do focinho até a linha vertical, passando pelo início da nadadeira anal. $\mathrm{O}$ desenvolvimento morfométrico foi analisado através de regressão simples, aplicada aos dados logaritimizados aos quais foram ajustados à equação $y=a x^{b}$ para obtenção dos valores de $b$, que indicam o tipo de relação entre as variáveis ( $b>1$ - alometria positiva; $b<1>0$ - alometria negativa; $b=1$ - isometria) (PERES-Neto 1995). Para as análises de relações corporais, as variáveis morfométricas foram relacionadas com o comprimento padrão e da cabeça ao longo do desenvolvimento larval. As relações corporais para a altura do corpo, comprimento da cabeça e diâmetro do olho foram estabelecidas utilizando-se os critérios sugeridos por LEIS \& TRNSKY (1989).

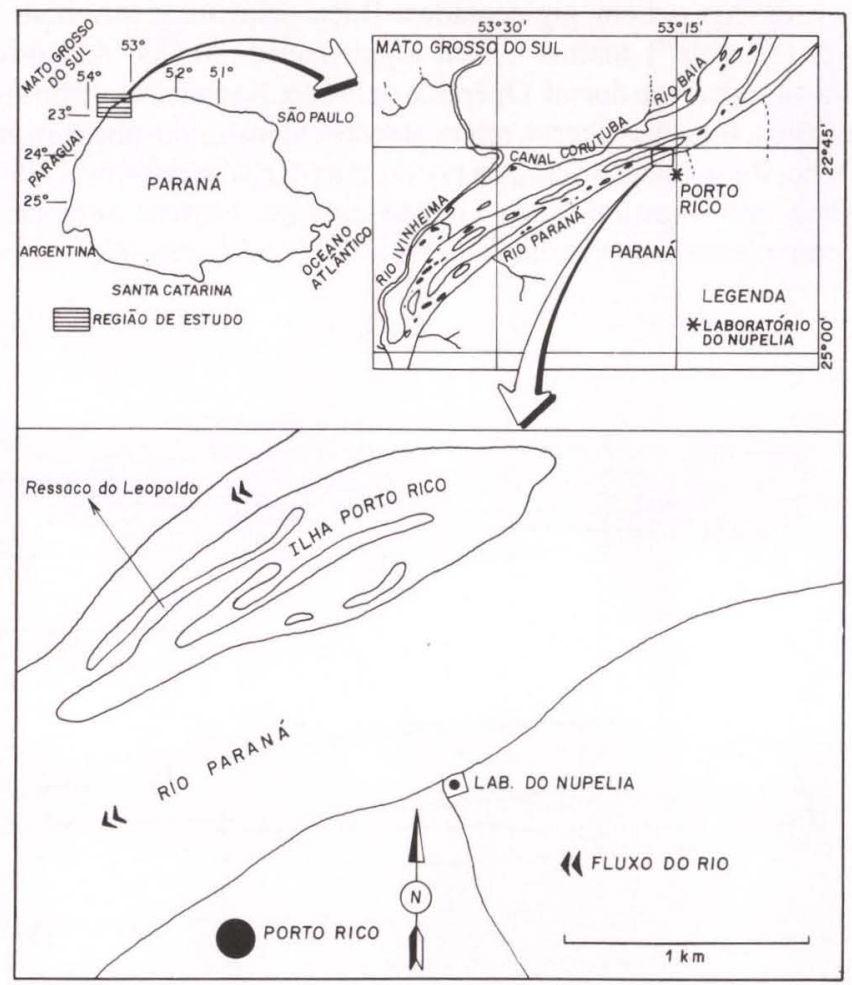

Fig. 1. Localização da estação de amostragem.

Para a análise da distribuição temporal, as larvas e juvenis de Apareiodon affinis capturados foram padronizadas para um volume de $10 \mathrm{~m}^{3}$ de água filtrada segundo TANAKA (1973), modificado.

A densidade relativa de organismos (ind. $/ 10 \mathrm{~m}^{3}$ ), foi obtida através da seguinte expressão: $\mathbf{D}=\mathbf{C} / \mathbf{B}$, onde: $(\mathbf{D})$ densidade relativa de indivíduos, $(\mathbf{C})$ número total de indivíduos coletados, (B) número de meses ou horários com ocorrência de organismos. 


\section{RESULTADOS E DISCUSSÃO}

\section{Caracterização morfológica}

Durante o período de estudo foram obtidos 53 indivíduos em estágio de pré-flexão, 25 em flexão, 67 em pós-flexão, 8 juvenis, e nenhum recém-eclodido. A descrição de cada estágio é apresentada a seguir:

\section{Período Larval}

\section{Estágio de Pré-flexão}

Larvas neste estágio apresentaram o comprimento padrão variando de 4,46 a $7,50 \mathrm{~mm}(\bar{x}=5,77 \pm 0,73)$. Notocorda não flexionada e visível por transparência. Os olhos são esféricos e bem pigmentados. Boca pequena e terminal. Membrana embrionária ("finfold") hialina e sem pigmentação. Bexiga natatória inflada e pigmentada na superfície dorsal. Opérculo definido. Nadadeiras peitorais formadas, porém sem raios. Intestino aberto, relativamente extenso, com abertura anal no terço final do corpo. Pigmentação formada por cromatóforos puntiformes que se concentram ao longo do intestino. Saco vitelino ausente. Narinas simples. Miômeros evidentes com número total variando de 35 a 39 ( 27 a 31 pré e 8 a 11 pós-anal) (Fig. 2A).
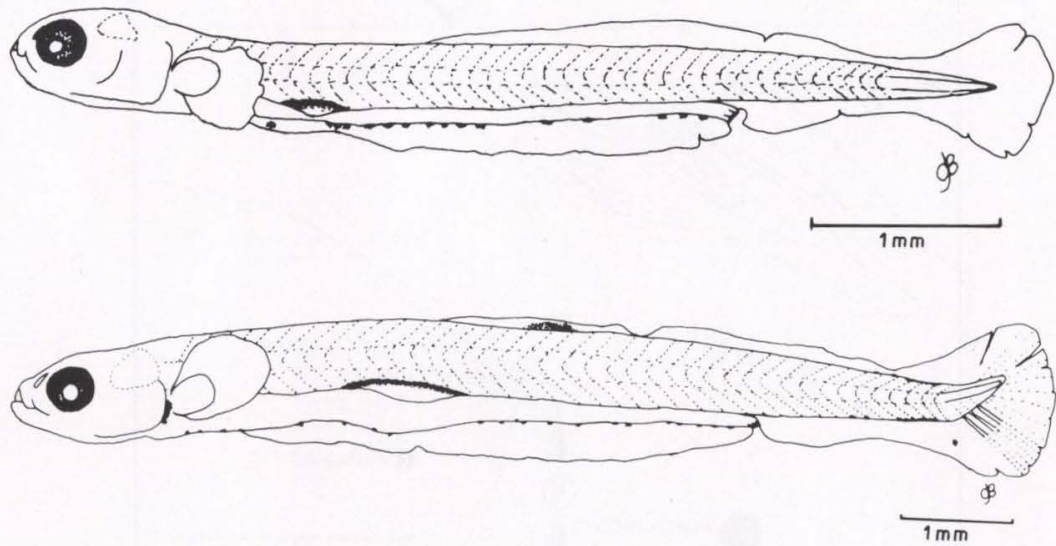

B

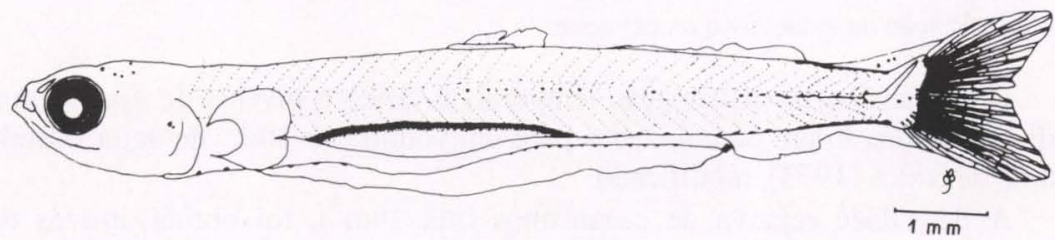

Fig. 2. Larvas de Apareiodon affinis com 5, 23mm CP (A), 8, 91 mm CP (B) e 9,68mm CP (C). 


\section{Estágio de Flexão}

Os indivíduos neste estágio apresentaram comprimento padrão variando de 7,38 a $12,43 \mathrm{~mm}(\overline{\mathrm{x}}=9,86 \pm 1,18)$. Miômeros evidentes, variando de 37 a 40 (29 a 32 pré e 7 a 10 pós-anal). Afim de melhor vizualizar o desenvolvimento morfológico, as larvas foram divididas em duas fases de acordo com a sua evolução.

Fase 1. Delineamento da nadadeira dorsal na membrana embrionária (aproximadamente $8,91 \mathrm{~mm}$ ). Caudal com alguns raios logo após a completa flexão da notocorda. Pigmentação escassa, restrita ao longo do intestino e pedúnculo caudal. Membrana embrionária presente. Boca terminal. Narina simples. Bexiga natatória inflada com pigmentação na superfície dorsal (Fig. 2B).

Fase 2. Formação dos raios das nadadeiras dorsal e anal (com cerca de $9,68 \mathrm{~mm}$ ). Caudal com a maioria dos raios formados, sendo alguns segmentados. Pigmentação formada por cromatóforos puntiformes presentes ao longo do intestino, na parte superior da cabeça, linha lateral, pedúnculo caudal e entre os raios da nadadeira caudal. Bexiga natatória inflada com pigmentação na superfície dorsal (Fig. 2C).

\section{Estágio de Pós-flexão}

Neste estágio o comprimento padrão variou entre 10,14 e $14,29 \mathrm{~mm}$ $(\overline{\mathrm{x}}=12,27 \pm 0,85)$. O número total de miômeros ficou entre 37 e 42 ( 28 a 31 pré e 7 a 11 pós-anal). A exemplo do anterior, este estágio também foi dividido em duas fases:

Fase 1. Os raios das nadadeiras dorsal e anal encontram-se em formação (aproximadamente $11,00 \mathrm{~mm}$ ). Formação da nadadeira adiposa. Aparecimento do botão da nadadeira pélvica. Bexiga natatória e notocorda ainda são visíveis por transparência. Cromatóforos puntiformes presentes na nadadeira caudal, pedúnculo caudal e parte superior da cabeça (Fig. 3A).

Fase 2. As nadadeiras pélvicas, anal, dorsal e caudal encontram-se formadas e com os raios segmentados (aproximadamente 13,14mm). A nadadeira peitoral com alguns raios formados. Pigmentação é mais intensa que os estágios anteriores, principalmente na região da boca, e entre os raios das nadadeiras dorsal e peitoral. Nadadeira adiposa formada. Membrana embrionária restrita à parte ventral do corpo. Notocorda e bexiga natatória não mais visualizadas, em função da cobertura pela epiderme (Fig. 3B).

\section{Periodo Juvenil}

Neste período o comprimento padrão variou de 13,43 a $24,00 \mathrm{~mm}$ $(\bar{x}=16,93 \pm 3,12)$. Os exemplares apresentaram características de um adulto. Pigmentação formada por cromatóforos puntiformes que se distribuem-se irregularmente na cabeça, boca, linha lateral e região dorsal do corpo. Pequenos cromatóforos estão presentes entre os raios das nadadeiras. Formação completa das nadadeiras, inclusive com segmentação de raios. Raios dendríticos na caudal. Número total: P. 8-10, V. 6-8, D. 10-12, e A. 6-9. Narinas duplas. Escamas presentes. Boca inferior (com aproximadamente $18,00 \mathrm{~mm}$ ) (Fig. 3C).

Durante a análise do desenvolvimento morfométrico de $A$. affinis, observouse que este é semelhante ao que foi obtido por OLDANI (1977) no médio rio Paraná. 

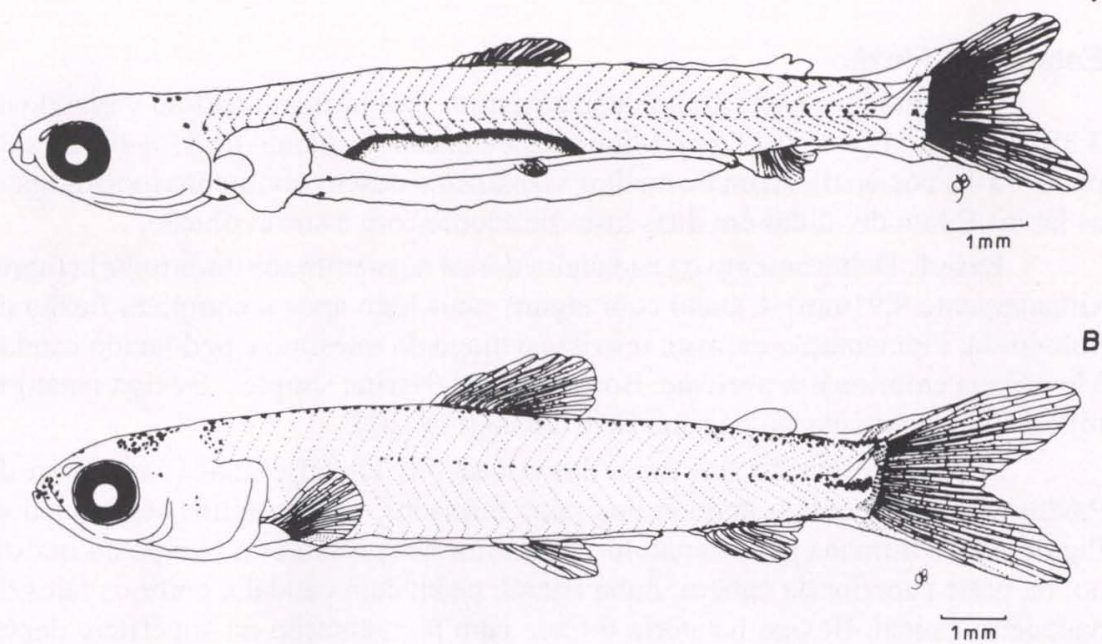

B

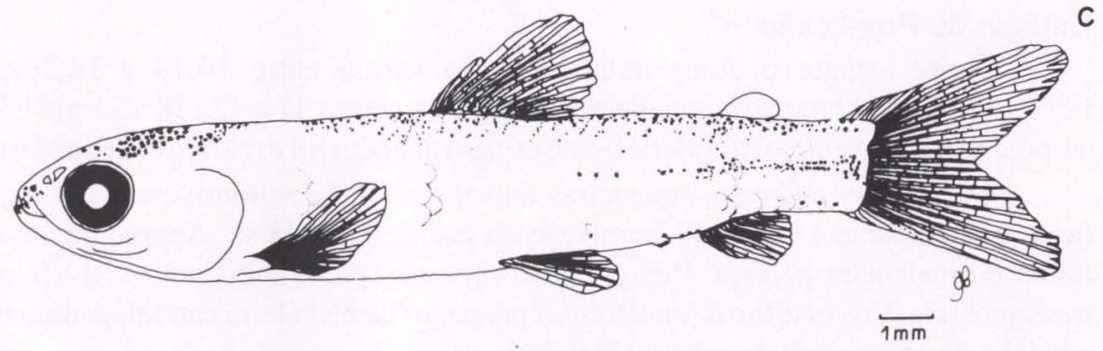

Fig. 3. Larvas de Apareiodon affinis com 11,00mm CP (A), 13,14mm CP (B) e juvenil com $16,00 \mathrm{~mm}$ CP.

O número total de miômeros (39-42), corresponde ao número de vértebras encontradas por este autor que foi de 39-41. Segundo LAUDER JR. (1980), o número de vértebras acrescido de uma unidade, apresenta uma correspondência como o número de miômeros das larvas, podendo ajudar na identificação da espécie. Ressalta-se que esse caracter deve ser apenas auxiliar, devendo sempre estar associado a outros, como pigmentação, tamanho e estágios de desenvolvimento, uma vez que muitas vezes ocorre sobreposição do número de vértebras entre espécies semelhantes.

A seqüência de formação das nadadeiras segue o mesmo padrão de outros Characiformes, como Mylossoma paraguayensis (Norman, 1929) (OLDANI 1983); Semaprochilodus insignis (Schomburgk, 1841) (ARAúJO-LIMA 1985) e para Prochilodus scrofa (Valenciennes, 1836) (CAVICCHIOLI \& LEONHARDT 1993). Outra característica importante observada nesta espécie é a posição da boca, que nos primeiros estágios de desenvolvimento é terminal, passando a inferior no período juvenil. Essa mudança também foi observada por SAZIMA (1980), em Apareiodon piracicabae (Eigenmann \& Ogle, 1907), parecendo ser esta uma característica do gênero. 


\section{Relações Corporais}

As variáveis morfométricas foram relacionados com o comprimento padrão e o comprimento da cabeça ao longo do desenvolvimento (Fig. 4A-G).

A análise das relações corporais mostraram que as larvas sofreram grandes variações na altura do corpo, passando de muito longa $(8,90 \%)$ no período de pré flexão, a longa nos períodos de flexão (10,51\%), pós-flexão $(13,65 \%)$ e juvenil $(17,01 \%)$ (Fig. 4A); o comprimento da cabeça é pequeno nos estágios de pré-flexão $(9,26 \%)$ e flexão (17,23\%), e moderado em pós-flexão (22,54\%) e juvenil $(26,08 \%)$ (Fig. 4B); enquanto a distância pré-anal apresentou variação de $73,47 \%$ a $83,16 \%$ (Fig. 4C), e a pré-dorsal de $41,47 \%$ a $49,48 \%$ (Fig. 4D), todas relacionadas ao comprimento padrão. Em relação ao comprimento da cabeça, o diâmetro do olho é grande apenas no período de pré-flexão $(34,46 \%)$, passando a moderado nos estágios de flexão (32,89\%), pós-flexão (28,31\%) e juvenil (26,78\%) (Fig. 4E); a altura da cabeça e o comprimento do focinho também mostraram variações marcantes durante o desenvolvimento ontogênico. O comprimento do focinho variou de $10,00 \%$ a $29,25 \%$ (Fig. 4 F), enquanto que a altura da cabeça, variou de $44,44 \%$ a $67,60 \%$ (Fig. $4 \mathrm{G}$ ).

As mudanças observadas nas relações corporais nos estágios de pré-flexão e flexão, principalmente na altura da cabeça e no diâmetro do olho, podem estar relacionada com o desenvolvimento da cabeça. CAVICCHIOLI \& LEONHARDT (1993), estudando o desenvolvimento morfométrico de $P$. scrofa, sugerem que esse aumento inicial da cabeça pode indicar um desenvolvimento na parte cerebral das larvas, podendo levar a diversificação das habilidades motoras, sensoriais e mudanças de comportamento, tipo de habitat e alimentação, o que provavelmente, explicaria os resultados obtidos na variação nictemeral, quando as larvas apresentaram comportamento diferenciado de acordo com o estágio de desenvolvimento (Fig. 7).

\section{Desenvolvimento morfométrico}

Conforme demonstrado na tabela I, esta espécie apresentou alometria positiva (b>1) somente para o comprimento da cabeça em relação ao comprimento padrão, ou seja esta variável apresenta crescimento proporcionalmente maior do que o comprimento padrão, entretanto o valor de b muito próximo a 1 , sugere quase uma isometria, o que poderia explicar o valor de "t" não significativo entre estas variáveis. Com relação as demais variáveis, a alometria foi negativa $(b<1)$, demostrando um crescimento proporcionalmente menor da altura do corpo, distâncias pré-anal e pré-dorsal em relação ao comprimento do corpo.

As relações entre o diâmetro do olho, altura da cabeça e comprimento do focinho em relação ao comprimento da cabeça, foram alométricas negativas, indicando que além do incremento no próprio comprimento da cabeça em relação ao comprimento padrão (alometria positiva), as partes da cabeça alteram-se de maneira desigual. WiLhelm (1984), estudando as diferenças no crescimento da cabeça de três espécies de ciclídeos do gênero Haplochromis (Hilgendorf, 1888), sugeriu que cada estrutura, ao longo da ontogenia, exerce um efeito diferente na posição e tamanho de outra estrutura. 

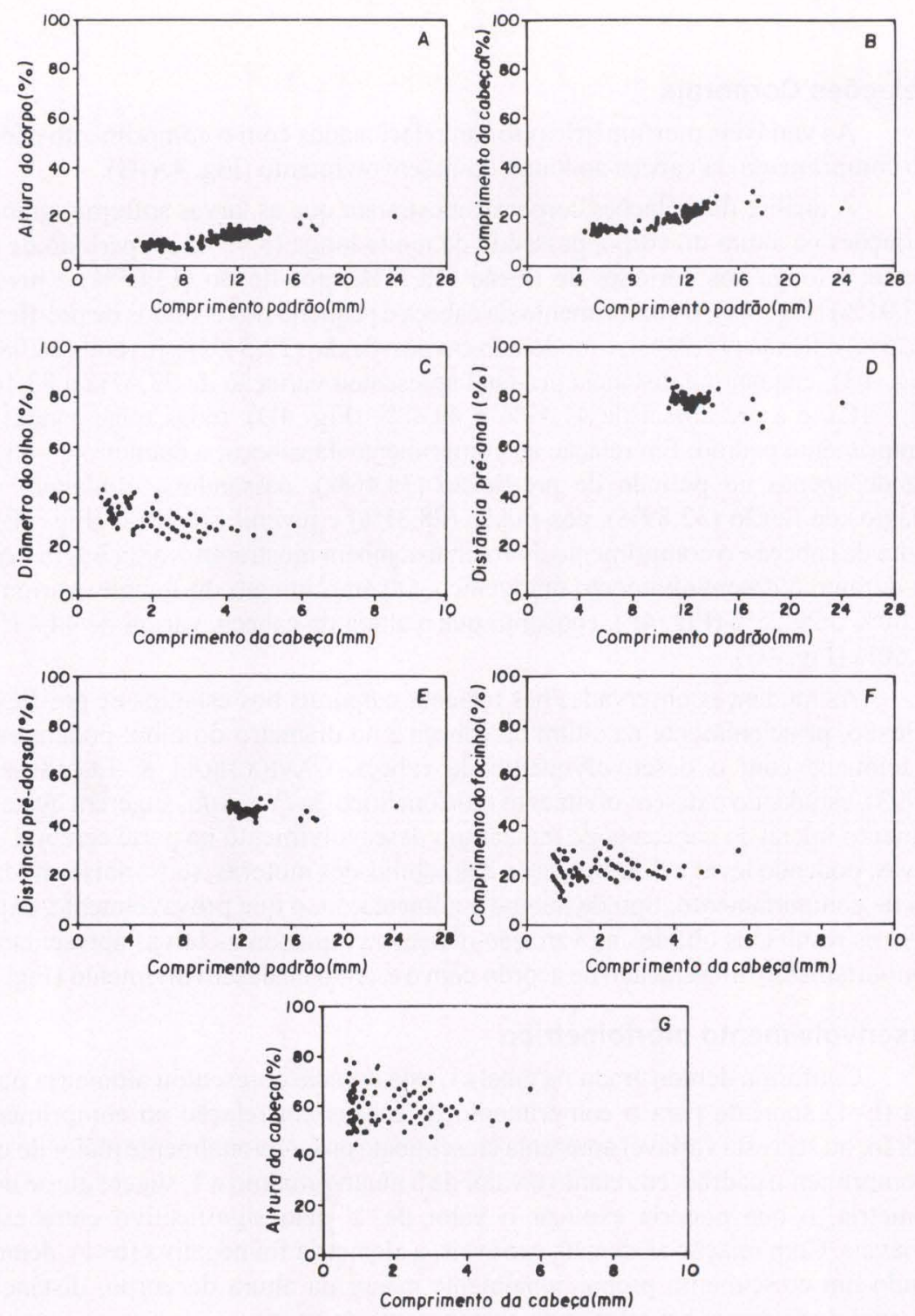

Fig. 4. Relações corporais entre A) altura do corpo, B) comprimento da cabeça, C) distâncias pré-anal e D) pré-dorsal em relação ao comprimento padrão e E) diâmetro do olho, F) altura da cabeça e G) comprimento do focinho em relação ao comprimento da cabeça para larvas e juvenis de Apareiodon affinis.

\section{Distribuição Temporal}

Durante o período, foram verificadas capturas de larvas entre agosto/1991 e fevereiro/1992 (Fig. 5). As maiores densidades ocorreram em novembro/1991 $\left(12,35 \mathrm{ind} / 10 \mathrm{~m}^{3}\right)$, seguido de dezembro/1991 e fevereiro/1992, (4,04 e 2,05 
ind $/ 10 \mathrm{~m}^{3}$, respectivamente). A elevada captura de larvas e juvenis entre esses meses sugerindo ser este o período reprodutivo da espécie na região. Esses resultados são semelhantes àqueles obtidos por GoDoy (1975) no rio Mogi Guassu, onde a época reprodutiva estendeu-se de setembro a maio. Segundo BARBIERI et al. (1983), esse extenso período reprodutivo pode estar relacionado com a desova parcelada apresentada pela espécie.
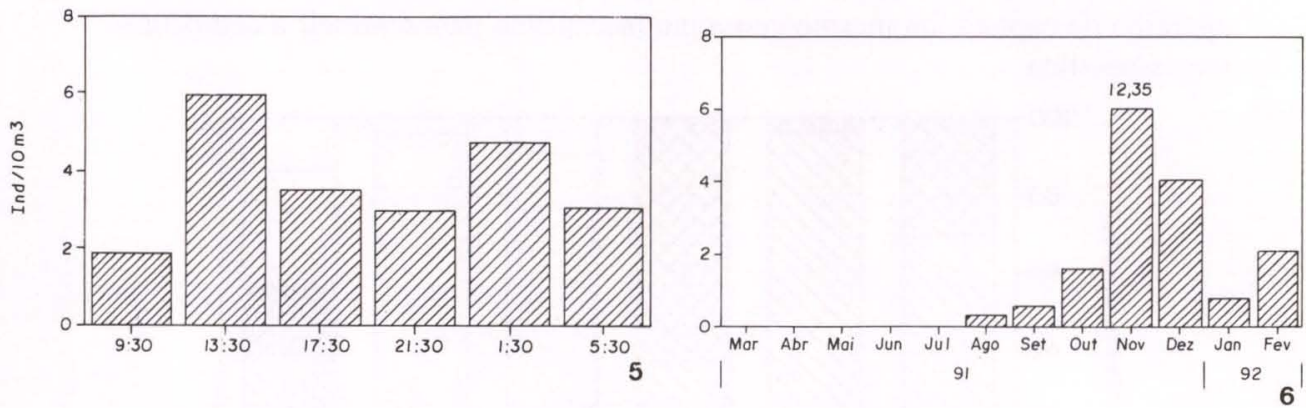

Figs 5-6. Densidades relativas mensais de Apareiodon affinis coletadas no Ressaco do Leopoldo. (5) Capturas mensais; (6) durante ciclos nictemerais.

Tabela I. Valores da equação potencial $\left(y=a x^{b}\right)$ e do $r^{2}$ (coeficiente de determinação), para os dados logaritimizados das variáveis morfométricas em relação ao comprimento padrão e da cabeça, para larvas e juvenis de Apareiodon affinis. (N) Número de exemplares, (a) coeficiente linear, (b) coeficiente alométrico, $(\mathrm{P})$ probabilidade, (ns) não significativo.

\begin{tabular}{lcrccc}
\hline Relação & $N$ & $a( \pm S D a)$ & $B( \pm S D b)$ & $r^{2}$ & $P$ \\
\hline CP $\times$ ACO & 153 & $0,28 \pm 0,02$ & $0,87 \pm 0,02$ & 0,93 & $p<0,001$ \\
CP $\times$ CC & 153 & $0,26 \pm 0,02$ & $1,03 \pm 0,02$ & 0,95 & $n s$ \\
CP $\times$ FNA & 83 & $-1,07 \pm 0,03$ & $0,89 \pm 0,03$ & 0,93 & $p<0,001$ \\
CP $\times$ FND & 65 & $0,69 \pm 0,04$ & $0,88 \pm 0,03$ & 0,91 & $\mathrm{p}<0,001$ \\
CC $\times$ DOL & 151 & $0,98 \pm 0,01$ & $0,46 \pm 0,01$ & 0,94 & $\mathrm{p}<0,001$ \\
CC $\times$ AC & 151 & $0,91 \pm 0,01$ & $0,80 \pm 0,02$ & 0,93 & $p<0,001$ \\
CC $\times$ CF & 153 & $0,89 \pm 0,01$ & $0,46 \pm 0,11$ & 0,93 & $p<0,001$ \\
\hline
\end{tabular}

As mudanças ambientais ocorridas neste período, como o início da elevação do nível fluviométrico (observação pessoal), provavelmente tenham influenciado a reprodução desta espécie. Segundo WELCOMME (1979), esse sincronismo entre o período reprodutivo e as cheias visa assegurar quali e quantitativamente a máxima disponibilidade de alimento às fases iniciais de desenvolvimento, propiciando um rápido crescimento e ultrapassando os estágios vulneráveis à predação mais intensa.

Quanto a análise da variação nictemeral constatou-se que a espécie não apresentou um padrão definido de captura, sendo encontrada em todos os horários amostrados. A diferença entre os períodos diurno e noturno não foi relevante, com maior densidade registrada às $13 \mathrm{~h} 30 \mathrm{~min}\left(5,94 \mathrm{ind} / 10 \mathrm{~m}^{3}\right)$, seguida da $01 \mathrm{~h} 30 \mathrm{~min}$ $\left(4,70 \mathrm{ind} / 10 \mathrm{~m}^{3}\right)$ (Fig. 6). 
A variação nictemeral, por estágios de desenvolvimento, mostra que durante o período diurno foram capturadas somente larvas em pré-flexão e flexão, sendo o contrário observado para os estágios mais avançados (pós-flexão e juvenil), onde as maiores capturas foram obtidas durante a noite (Fig. 7).

As elevadas capturas de larvas de $A$. affinis no período diurno podem estar associadas às formas iniciais de desenvolvimento (pré-flexão e flexão), cujas larvas apresentam pouca acuidade visual e baixa capacidade de natação para evitar o aparelho de captura, ou mesmo como um mecanismo para diminuir a competição intraespecífica.

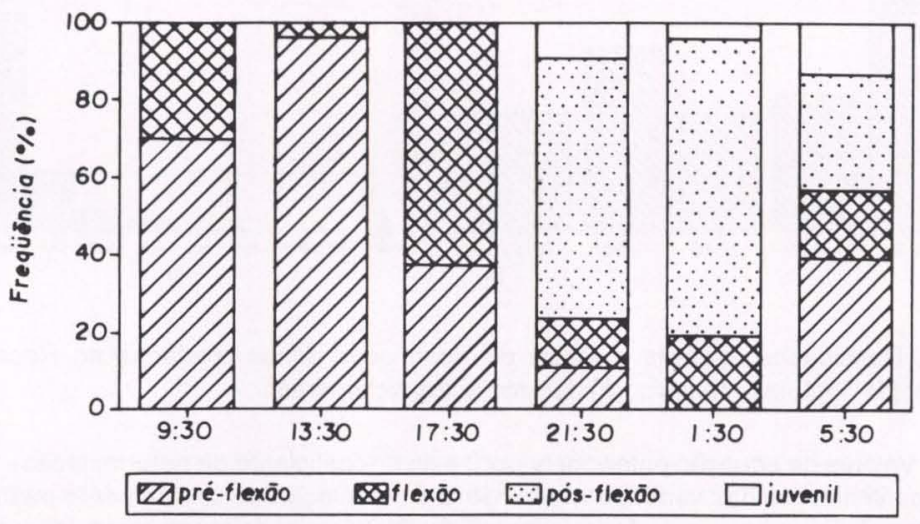

Fig. 7. Frequência de captura por estagios de desenvolvimento de Apareiodon affinis, durante os ciclos nictemerais, no ressaco do Leopoldo.

AGRADECIMENTOS. Os autores agradecem ao Núcleo de Pesquisas em Limnologia, Ictiologia e Aqüicultura (Nupélia), pelo apoio logístico durante o período de coleta, ao desenhista Jaime Luiz Lopes Pereira pela confecção dos gráficos e ao Prof. Msc. Luiz Carlos Gomes pelas valiosas sugestões.

\section{REFERÊNCIAS BIBLIOGRÁFICAS}

Ahlstrom, E.A. \& O.P. BALl. 1954. Description of eggs and larvae of jack mackarel (Trachurus symetricus) and distribuition and abundance of larvae in 1950 and 1951. Fish. Bull. 56: 209-245.

AHLstRom, E.A. \& H.G. Moser. 1976. Eggs and larvae of fishes and their role in systematic investigations and in fisheries. Rev. Trav. Inst. Peches. Marit., Nantes, 40 (3): 379-398.

ARAÚJo-LiMA, C.A.R.M. 1985. Aspectos biológicos de peixes amazônicos. V. Desenvolvimento larval do jaraqui-escama grossa, Semaprochilodus insignis (Characiformes, Pisces) da Amazônia Central. Rev. Brasil. Biol., Rio de Janeiro, 45 (4): 423-431.

BARBIERI, G.; J.R. VERANI \& M.C. BARBIERI. 1983. Análise do comportamento reprodutivo de Apareiodon affinis (Staindachner, 1879), Apareiodon ibitiensis (Campos, 1944) e Parodon tortuosus (Eigenmann \& Norris, 1900) do rio Passa 
Cinco, Ipeúna, SP (Pisces, Parodontidae). Anais do III Seminário Regional de Ecologia, São Carlos, p. 189-199.

CAVICCHIOLI, M. \& J.H. LEONHARDT. 1993. Estudo do desenvolvimento morfológico de larvas de curimbatá, Prochilodus scrofa (Steindachner, 1882), obtidas de reprodução induzida. Rev. Unimar, Maringá, 15 (Supl.): 109-124.

Fuiman, L.A.; J.V. CONNER; B.F. LATHROP; G.L. BuYNAK; D.E. SNYDER \& J.J. Loos. 1983. State of the art of identification for cyprinid fish larvae from easter north America. Trans. Amer. Fish. Soc. 112: 319-332.

Godoy, M.P. 1975. Peixes do Brasil Sub-ordem Characoidei- Bacia do rio Mogi Guassu. Piracicaba, Franciscana, vol. 3, p.400-627.

Kendall, A.W.; E.H. Ahlstrom \& H.G. Moser. 1984. Early life history stages of fishes and their characters. Spec. publ. Amer. Soc. Ichthyl. Herpetol. 1: $11-22$

LAUDER JR., G.V. 1980. On the relationship of the myotome to the axial skeleton in vertebrate evolution. Paleobiology 6: 51-56.

LEIS, J.M. \& T. TRNSKI. 1989. The larvae of Indo-Pacific shorefishes. Honolulu, University of Hawaii Press, $371 \mathrm{p}$.

Peres-Neto, P.R. 1995. Introdução a análises morofométricas, p. 57-89. In: P.R. Peres-Neto; J.L. Valentin \& F.A.S. Fernandez (Eds). Tópicos em tratamentos de dados biológicos. Rio de Janeiro, Univ. Federal do Rio de Janeiro, $161 \mathrm{p}$.

OLDANI, N.O. 1977. Identificación y morfologia de larvas de Apareiodon affinis (Steindachner, 1879) (Pisces, Parodontidae). Physis 37 (93): 133-140. . 1983. Identificación y morfologia das larvas, juveniles y adultos de Mylossoma paraguayensis, N. 1929 (Pisces, Characidae). Studies Neotrop. Fauna Envirom. 18 (2): 89-100.

SAZIMA, I. 1980. Behavior of two brazilian species of parodontid fishes, Apareiodon piracicabae and A. ibitiensis. Copeia 1: 169-171.

SNYDER, D.E. 1981. Contributions to a guide to the cypriniform fish larvae of the upper Colorado river system in Colorado. Denver, United States Bureau of Land Management, 81p.

TANAKA, S. 1973. Stock assesment by means of ichtyoplankton surveys. FAO Fish Tech Pap. 122: 33-51.

VAZZOLER, A.E.A.M. 1996. Biologia da reprodução de peixes teleósteos: teoria e prática. Maringá, Eduem, SBI, XVIII+169p.

WELCOMME, R.L. 1979. Fisheries ecology of floodplain rivers. London, Logman, $317 \mathrm{p}$.

WILHELM, W. 1984. Interspecific allometric growth differences in the head of three haplochromine species (Pisces, Cichlidae). Netherl. Jour. Zool. 34 (4): 622628.

Recebido em 05.VI.1997; aceito em 25.XI.1998. 